\title{
Solar cosmic rays during the extremely high ground level enhancement on 23 February 1956
}

\author{
A. Belov ${ }^{1}$, E. Eroshenko ${ }^{1}$, H. Mavromichalaki ${ }^{2}$, C. Plainaki ${ }^{2}$, and V. Yanke $^{1}$ \\ ${ }^{1}$ Institute of Terrestrial Magnetism, Ionosphere and Radio Wave Propagation (IZMIRAN), 42092, Troitsk, Moscow Region, \\ Russia \\ ${ }^{2}$ Nuclear and Particle Physics Section, Physics Department, Athens University Pan/polis-Zografos 15771 Athens, Greece
}

Received: 21 January 2005 - Revised: 12 May 2005 - Accepted: 25 May 2005 - Published: 15 September 2005

\begin{abstract}
The 23 February 1956 ground level enhancement of the solar cosmic ray intensity (GLE05) is the most famous among the proton events observed since 1942. But we do not have a great deal of information on this event due to the absence of solar wind and interplanetary magnetic field measurements at that time. Furthermore, there were no X-Ray or gamma observations and the information on the associated flare is limited. Cosmic ray data was obtained exclusively by ground level detectors of small size and in some cases of a non-standard design. In the present work all available data from neutron monitors operating in 1956 were analyzed, in order to develop a model of the solar cosmic ray behavior during the event. The time-dependent characteristics of the cosmic ray energy spectrum, cosmic ray anisotropy, and differential and integral fluxes have been evaluated utilizing different isotropic and anisotropic models. It is shown that the most outstanding features of this proton enhancement were a narrow and extremely intense beam of ultra-relativistic particles arriving at Earth just after the onset and the unusually high maximum solar particle energy. However, the contribution of this beam to the overall solar particle density and fluency was not significant because of its very short duration and small width. Our estimate of the integral flux for particles with energies over $100 \mathrm{MeV}$ places this event above all subsequent. Perhaps the number of accelerated low energy particles was closer to a record value, but these particles passed mainly to the west of Earth.

Many features of this GLE are apparently explained by the peculiarity of the particle interplanetary propagation from a remote (near the limb) source. The quality of the available neutron monitor data does not allow us to be certain of some details; these may be cleared up by the incorporation into the analysis of data from muonic telescopes and ionization chambers operating at that time.
\end{abstract}

Keywords. Interplanatary physics (Cosmic rays; Energetic particles) - Solar physics, astrophysics and astronomy (Flares and mass injections)

Correspondence to: H. Mavromichalaki

(emavromi@cc.uoa.gr)

\section{Introduction}

Although 64 GLEs have been recorded since 1956, all of them rank below the 1956 event by more than an order of magnitude. The superiority of this event is seen well in Fig. 1, where the biggest changes in the neutron monitor counting rates during proton events in the 19th, 22nd, and 23rd solar cycles are plotted as if they started at the same time. A difference between these events is even more impressive if one remembers that the South Pole station (with geomagnetic cutoff rigidity about $0 \mathrm{GV}$ and located at $2800 \mathrm{~m}$ above the sea level) has a much better sensitivity for solar proton observations in comparison with Leeds (cutoff rigidity $\sim 2.2 \mathrm{GV}$ and located near sea level), which recorded the maximum effect in February 1956, as much as $\sim 4500 \%$.

The event on 23 February exerted essential influence on the development of solar-terrestrial physics. It brought widespread attention to the Sun and cosmic ray observations, promoted the organization and success of cosmic radiation studies during the IGY and led to the creation of the world-wide cosmic ray observatory network. In the first few years after this event, numerous scientific analyses were published (Meyer et al., 1956; Sarabhai et al., 1956; Dorman, 1957; Van Allen and Winckler, 1957; Pfotzer, 1958). Many observations and results were combined and published in the Dorman monograph (1957). While many reviews and publications on solar cosmic rays (e.g. Dorman, 1963; Sandstrom, 1965; Dorman and Miroshnichenko, 1968; Akasofu and Chapman, 1972; Miroshnichenko, 1970; Miroshnichenko, 2001; Heristchi et al., 1976, Smart and Shea, 2002) discussed or mentioned this proton event, no one has yet conducted a detailed analysis using modern techniques and contemporary knowledge. The only exception was a paper by Smart and Shea (1990), where they constructed a model of the behavior of the high energy solar particles flux and pitch angle distributions using neutron monitor data.

Despite all of these papers, we should recognize that until now we know much less about the 23 February 1956 event than about later events. This restricts the possibility of deriving a generalized model of solar proton events, since we 


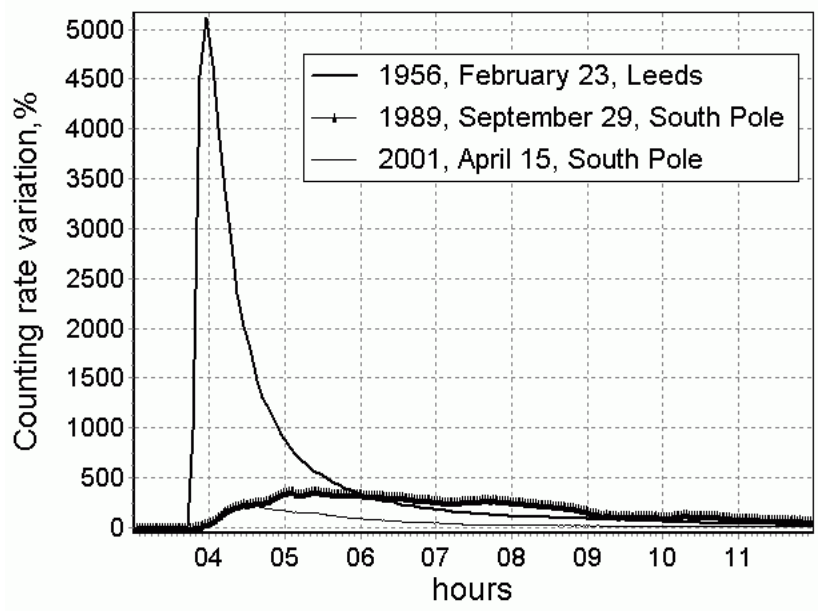

Fig. 1. Counting rate variations for the largest GLEs in different solar cycles.

cannot compare this event, which was the largest, with the more modest events that have occurred since 1956. All future proton events will inevitably be compared with this one, and to make the most of such a comparison we need to derive as much as possible from the existing data. This analysis will have bearing for our conclusions on the radiation hazards of solar comic rays and our estimations for space weather tasks.

In this paper data from thirteen neutron monitors were incorporated into a comprehensive analysis of this event, in order to define a more precise model for the behavior of solar cosmic rays. Some results on this study were also elucidated in Belov et al. (2004).

\section{Solar and geomagnetic activity during 1956}

The year 1956 belongs to the ascending phase of solar cycle 19, after a deep minimum of solar activity which took place in 1954. Over the 15 months between October 1953 and December 1954, the monthly average sunspot number remained less than 10. New sunspots began to be generated faster towards the end of 1955. In January-February 1956 the sunspot number became about 120 , which is a typical value for periods of high solar activity. However, some time later in solar cycle 19 much larger sunspot numbers were observed: in October 1957 the monthly average value reached 254. The GLE on 23 February 1956 (GLE 05) was the first ground level proton enhancement in the cycle. Later, in other cycles some GLEs were observed even at the earlier stage of the ascending phase of solar activity (for example, GLE28 and GLE29 in September 1977, or GLE55 at the beginning of November 1997), but all of them were significantly smaller.

The event on February 1956 occurred in sunspot group 17351 (Greenwich catalogue), which was located in the northern portion of the solar disk (N22) and on 16 February 1956 had a size that exceeded 1700 millionths of the solar surface. This group evolved quickly in January, reaching a

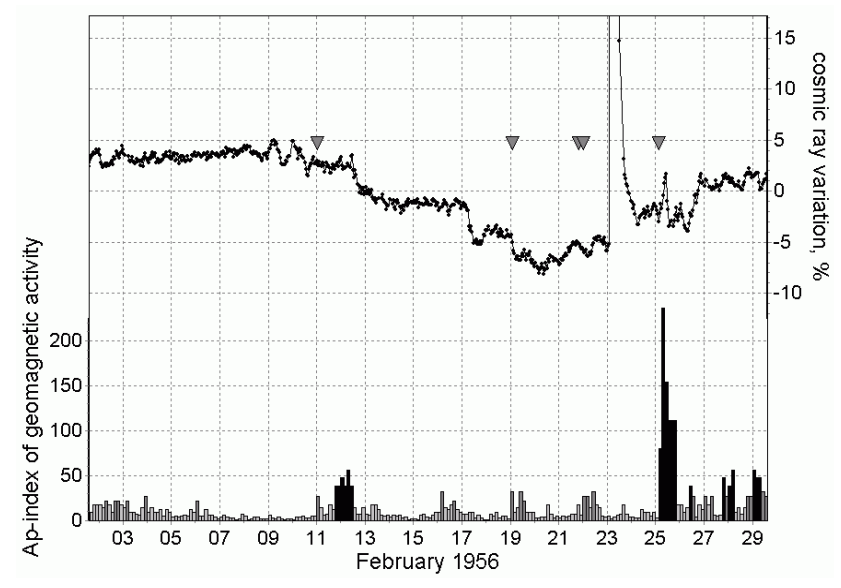

Fig. 2. Counting rate variations at Climax neutron monitor and Ap index of geomagnetic activity in February 1956. Great fluxes on 23 February are not plotted. Triangles at the top of the figure correspond to the SSC.

size of 1958 millionths of the solar sufrace and therefore was the largest sunspot group in this period. From 12 to 26 February another sunspot group of a smaller size, 17353, crossed the solar disk. It appeared almost at the same heliolongitude area as the 17351 sunspot group, but in the Southern Hemisphere. This group reached a big square only in February, whereas in March it appeared already much smaller. The size of sunspot group 17351 was reduced later on but the group was observable until June. It is noteworthy that the maximum of flare activity for the sunspot group 17351 manifested during the period when both AR 17351 and AR 17353 were very large and were close to their peak sizes.

Flare activity increased at the end of October 1955 and in January 1956. The majority of the large January flares were related to the sunspot group which became AR 17351 . From 13 to 20 February, twelve optical flares were generated by group 17351, the most significant occurring on 14, 17 and 21 February. Large flares were also produced by group 17353. It is interesting that on 21 February another sunspot group, 17349 , located very close to AR 17351 was also activated, producing a 3-B flare at N40 latitude near the limb. The most important flare occurred when group 17351 was passing over the limb. This 3-B importance flare started on 23 February at 03:34 UT at coordinates N25W85 and produced the outstanding GLE 05. Since it was observed so close to the limb, we cannot exclude that it was partly behind the limb and could have started somewhat earlier.

In conjunction with minimum solar activity, the intensity of galactic cosmic rays peaked in 1954, and almost the same high level remained during 1955. A decrease in cosmic ray flux started in February 1956; for $10 \mathrm{GV}$ particles it reached $2.3 \%$ (Belov et al., 1997). However, the background of galactic cosmic rays was still close to the typical value obtained during the minimum of solar activity. A series of Forbush effects, possibly related to the activity of sunspot group 17351 , 
Table 1. Neutron monitor stations which data are employed in this analysis.

\begin{tabular}{llrrrrr}
\hline Station & Abbreviation & Lat $^{0}$ & Long & Alt $(m)$ & $\mathrm{H}_{o}(\mathrm{mb})$ & $\mathrm{Rc}(\mathrm{GV})$ \\
\hline Albuquerque & albq & 35.08 & -106.62 & 1567 & 800.0 & 4.47 \\
Arneb USS & arnb & -41.28 & 174.77 & 0 & 1013.0 & 3.45 \\
Berkley & berk & 37.87 & -122.30 & 0 & 1005.0 & 4.55 \\
Chicago & chgo & 41.83 & -87.67 & 49 & 1000.0 & 1.71 \\
Climax & clmx & 39.37 & -106.18 & 3400 & 667.0 & 3.06 \\
Gottingen & gott & 51.52 & 9.93 & 273 & 1013.0 & 3.00 \\
Huancayo & huan & -12.03 & -75.33 & 3400 & 704.0 & 13.44 \\
Leeds & leed & 53.80 & -1.55 & 100 & 1004.0 & 2.15 \\
Mexico City & mxco & 19.33 & -99.18 & 2274 & 779.0 & 9.74 \\
Mt. Norikura & mtnr & 36.11 & 137.55 & 2770 & 888.0 & 11.35 \\
Ottawa & otwa & 45.44 & -75.68 & 57 & 1008.0 & 1.08 \\
Sacramento Peak & sacp & 32.72 & -105.75 & 3000 & 680.0 & 5.10 \\
Stockholm & sthm & 59.35 & 17.95 & 0 & 1000.0 & 1.50 \\
Weissenau & weis & 47.80 & 9.50 & 427 & 960.0 & 4.08 \\
\hline
\end{tabular}

was recorded during the period before GLE05 (Fig. 2). The sequence of these Forbush decreases resulted in a total decrease of the galactic cosmic rays intensity by as much as $11 \%$ (Climax neutron monitor) by 20 February. A recovery started on 20-21 February and reached the value of $2.5 \%$ on 23 February. Interplanetary shocks arriving on 21 and 22 February did not lead to further decreases and did not even slow the recovery process. Just before GLE 05 the level of the galactic cosmic ray intensity was $8-9 \%$ lower than that in solar activity minimum.

Geomagnetic activity was relatively low in the time period 1953-1955. It increased slightly in January-February 1956 (the monthly average Ap was about 17.8 in January and about 15.4 in February). Eight magnetic storms took place in January 1956, but they all were minor or moderate. In February only one magnetic storm (on 11-12 February) was recorded before 25 February when the geomagnetic situation became moderately disturbed and the activity was a little above average. During the next three months the geomagnetic activity increased more strongly. Sudden storm commencements (SSC) occurred on 11, 19, 21 and 22 February, evidence of strong interplanetary disturbances. However, in the last three cases there were no magnetic storms. Large modulation effects in cosmic rays during 11-20 February confirm the significance of these interplanetary disturbances. The fact that magnetic storms did not evolve implies that the negative $B_{z}$ component of the IMF was small during these times. It is remarkable that in this case there were no magnetic storms following the central flares (for example, on 17 February), whereas after the flare on 23 February (near the limb) a magnetic storm did actually start. The shock arrival was registered on 25 February at 03:06 UT and it was a relatively fast interplanetary disturbance considering the remote western place of the source. This magnetic storm was the biggest one since April 1952, reaching the level of $K_{p}=8^{+}$.

\section{Data and methods}

As it was mentioned, there were no solar wind measurements at that time (and we can only guess about IMF properties), nor any information on CMEs; we have very limited information about the associated flare, without X-Ray and Gamma observations. The cosmic ray observations were carried out only by ground level detectors: standard and nonstandard neutron monitors, muonic telescopes and ionization chambers. The main advantage, which outweighs all possible minuses, is the magnitude of the enhancement. With this large magnitude, we can ignore the statistical accuracy of the cosmic ray detectors.

The majority of cosmic ray stations with standard neutron monitors appeared during the IGY period (Shea and Smart, 2000). Regular data for earlier years (pre-1956) can be found only for four neutron monitors. However, the GLE05 in February 1956 was recorded by 13 neutron monitors. The characteristics of these stations are taken from the old papers (Dorman, 1957; Shea and Smart, 2000; Yasue et al., 1982; Moraal et al., 2000) and presented in Table 1. Standard atmospheric pressure $\mathrm{H}_{0}$ was unknown as a rule. We present in Table 1 values of $\mathrm{H}_{0}$ taken from the later data publications, or estimated from the station altitude. In all cases where the geomagnetic cutoff rigidity of the station was unknown we have estimated its value by interpolation of the data presented in Shea et al. (1965) and Shea and Smart (1975). These neutron monitors were situated over a wide range of longitudes and covered a wide range of rigidities from $1 \mathrm{GV}(\mathrm{Ot}-$ tawa) to $13.45 \mathrm{GV}$ (Huancayo), with having rather satisfactory longitudinal overlapping due to their asymptotic directions (Fig. 3). Some of these data for the 23 February 1956 event were only in graphical form and it was impossible to find the original source of the data. But modern facilities and methods allow information on this outstanding event to be recovered. 


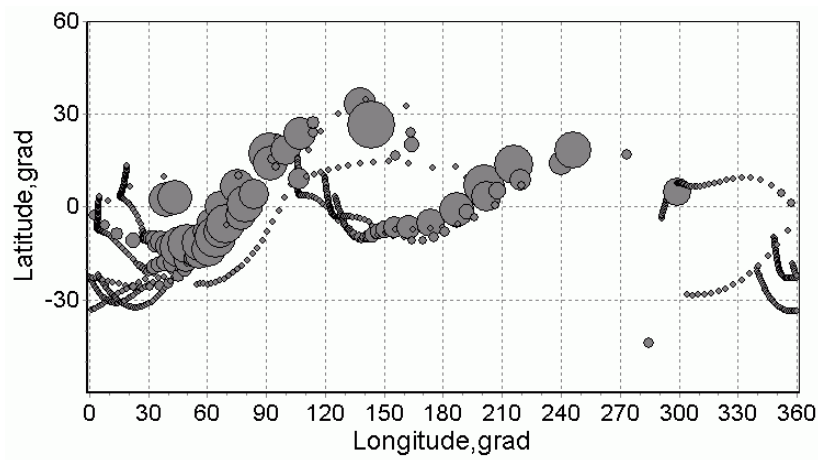

Fig. 3. Asymptotic directions for vertical incident particles for energy $<15 \mathrm{GeV}$ registered by neutron monitors available in February 1956. The circle radii correspond to the contribution of particles at fixed energy for power spectra with index -4 .

\subsection{Cosmic ray variation model}

Cosmic ray variations recorded by a ground level detector during a GLE may be written as follows (Dorman, 1963; Belov et al., 1994):

$\frac{\Delta N}{N_{0}}\left(t, t_{0}\right)=\frac{\int_{E_{c}}^{E_{u}} W\left(E, t_{0}, h\right) \frac{\Delta I}{I_{0}}(t, E) d E}{\int_{E_{c}}^{E_{u}} W\left(E, t_{0}, h\right) d E}$,

where $N_{0}$ is a basic counting rate resulting from a galactic cosmic ray flux $I_{0}$ and measured at the moment $t_{0} ; h_{i}$ is the atmospheric depth at the point of observation, $E_{c}$ is the least energy of the primary protons recorded by a detector, which corresponds to the threshold of geomagnetic cutoff rigidity $R c$ at the point of observation; $E_{u}$ is the upper energy limit for the solar particles registered in the event. Response functions $W\left(E, t_{0}, h\right)$ are given here as dependent on energy $E$ (but not on rigidity R) and $W\left(E, t_{0}, h\right) d E=W(R$, $\left.t_{0}, h\right)(d E / d R) d R$. Long-term variations and series of the Forbush decreases before this flare lead to the necessity of a correction in the response functions in the following way: $W\left(R, t_{0}, h\right)=W_{\min }(R, h)\left[1+\delta_{t 0}(R)\right]$. Here $W_{\min }(R, h)$ are the response functions in the solar activity minimum, and $\delta_{t 0}(R)$ is the variation of the primary spectrum of galactic cosmic rays from minimum activity to the base time $t_{0}$. Since there was no reliable data in that time to be sufficient for the estimation of the rigidity spectrum of galactic $\mathrm{CR}$ variations $\delta_{t 0}(R)$, we used a typical spectrum for long-term variations (Belov et al., 1994) as $a_{0} \frac{b_{w}+(10)^{\gamma_{0}}}{b_{w}+R^{\gamma_{0}}}$. In this formula $R$ is measured in GV, parameters $b_{w}=5$ and $\gamma_{0}=0.8$ are selected to fit this dependence closely to the rigidity spectrum typical for the Forbush decrease, and parameter $a_{0}=0.08$ is chosen to fit long-term variation at NM Climax. Since operating neutron monitors were at a variety of altitudes, the response functions $W_{\min }(R, h)$, taking into account the altitudinal dependence of the neutron component have been entered as:

$W_{\text {min }}(R, h)=\alpha \beta e^{-\alpha R^{-\beta}} R^{-(\beta+1)}$, where $\alpha=\exp (1.84+0.094 * h-0.09 * \exp (-11 * h) ; \beta=1.40-$ $0.56 * h+0.24 * \exp (-8.8 * h)$ ( $h$ in bars).

These functions have been used in many studies of galactic cosmic ray variations (Belov et al., 1997; Belov, 2000 and references) and in the analysis of GLEs (Belov et al., 1994; Belov and Eroshenko, 1996), mainly for the NM64 monitors. However, as it was shown in Clem and Dorman (2000), the difference between the response functions for IGY and NM64 neutron monitors is not significant. Equation (2) is in a good agreement with data on latitudinal surveys (Stoker et al., 1980; Villoresi et al., 2000) for rigidities >1.5-2 GV, but it seems to be inaccurate in the low energy range. In Belov and Struminsky (1997), the response functions within the low energy range were found to be close to a power law $E^{3.17}$ and this was used in our study for energies $\mathrm{E}<2 \mathrm{GeV}$ :

$W\left(E, t_{0}, h\right)=W\left(2 \mathrm{GeV}, t_{0}, h\right)(E / 2 \mathrm{GeV})^{3.17}$.

Cosmic ray intensity variations $\Delta I(t, E)$ in general are supposed to consist of two parts - isotropic $\Delta I_{0}$ and anisotropic part $\Delta I_{1}$ :

$\Delta I(t, E)=\Delta I_{0}+\Delta I_{1}+b_{0} f_{0}(E)+b_{1} f_{1}(E) \Psi_{1}(\chi, E),(4)$

where $\chi$ is the angular parameter of the solar CR anisotropy, $\Psi_{1}$ is the axis-symmetric function equal to 1 for $\chi=\chi_{0}$, and $b_{0}$ and $b_{1}$ are the magnitude of the isotropic and anisotropic parts of the enhancement.

To simplify the model and to reduce the number of unknown parameters we assumed that the shape and the direction of anisotropy are independent of energy. The energy dependence for isotropic and anisotropic parts is assumed to be the same, $\left(f_{0}(E)=f_{1}(E)\right)$. Several models have been sampled by their best fitting to neutron monitor data, to describe the spectrum and also the anisotropy of the solar cosmic rays during this event. For function $f_{0}$ eight different dependences have been checked: a) power law dependences by energy and rigidity $\left(\Delta I=E^{\gamma} ; \Delta I=R^{\gamma}\right)$, b) exponential dependences by energy and rigidity $\left(\Delta I=\exp \left(-E / E_{0}\right) ; \Delta I=\exp (-\right.$ $R / R_{0}$ ), c) power law and exponential dependences by $E$ for $\Delta I / I_{0}$ instead of $\Delta I$, d) power law and exponential dependences by $\mathrm{R}$ for $\Delta I / I_{0}$ instead of $\Delta I$. For the angular distribution function $\Psi_{1}$ three different models were checked:

$\Psi_{1}=\cos ^{n}\left(\chi-\chi_{0}\right)$,

$\left.\Psi_{1}=\exp \left(\left(\chi-\chi_{0}\right)^{2} / 2 \sigma_{1}^{2}\right)\right)$,

$\Psi_{1}=\exp \left\{-\left(n_{a} \sin \left(\chi-\chi_{0}\right)\right)^{2}\right\}$,

where $n_{0}$ in Eq. (5c) determines the width of the anisotropic flux: the more $n_{0}$ there is the more narrowing takes place in the anisotropic flux.

\subsection{Correction for the trend}

Since the GLE in 1956 was a long-lasting event we must correct the data for the trend (drift). At some high-latitude stations the enhancement was observed even until the last hours 


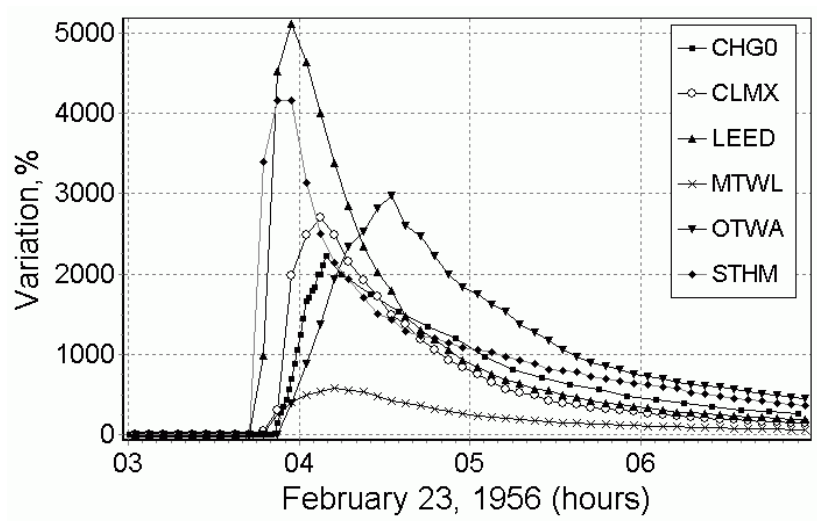

Fig. 4. Profiles of the cosmic ray enhancement recorded at different neutron monitors on 23 February 1956: CHGO - Chicago, CLMX Climax, LEED - Leeds, MTWL - Mt.Wellington, OTWA - Ottawa, STHM - Stockholm.

of 23 February. The level of the enhancement at these stations remained quite high (some \%), even $20 \mathrm{~h}$ after the onset. However, the counting rate of some low-latitude stations was continuing to rise due to the recovery of the GCR intensity after the series of Forbush effects. Therefore, data must be corrected for this trend, in order to study the time evolution of the solar cosmic rays (SCR). The trend of GCR in Climax data was found to be $0.083 \%$ /hour in assuming its almost linear dependence. To obtain the trend for other stations, the rigidity dependence of GCR variations was assumed as $\propto \mathrm{R}^{-0.8}$, which is sufficiently typical for the Forbush effect. Corrections for any other arbitrary station were calculated by means of response functions described above. None of these corrections exceeded $1.7 \%$, even at the end of 23 February.

\section{Results and discussion}

\subsection{Preliminary results}

It is possible to draw some preliminary conclusions even without complicated analysis of the data:

a) The enhancement on 23 February 1956 revealed itself to be extremely anisotropic. Counting rate variations plotted in Fig. 4 illustrate a large difference in the onsets at various stations. As one can see from Fig. 5, the observed anisotropy, as seen by the relative intensity variations between Leeds and Chicago during the first $10 \mathrm{~min}$, is almost $100 \%$. We come to the same conclusion when comparing the time profiles of $\mathrm{CR}$ variations in Fig. 4 recorded by the NMs in Leeds and Chicago. These two stations have similar cutoff rigidities $(2.15$ and $1.71 \mathrm{GV}$, respectively), but differ in longitude. If the flux is isotropic, then the flux increase should be larger at Chicago. However, the increase is observed to be higher at Leeds, and only beginning in the second hour after the onset does the counting rate become higher at Chicago. High anisotropy has been observed,

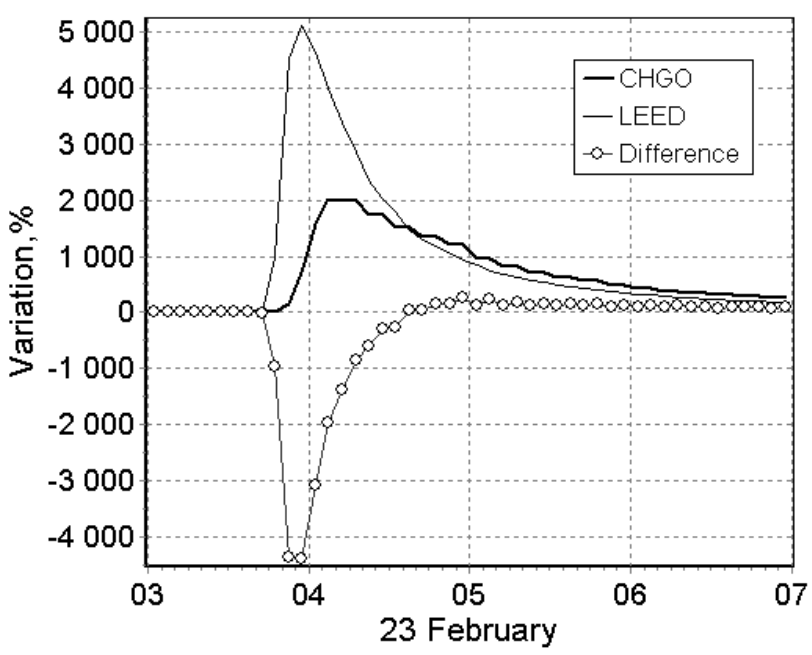

Fig. 5. Anisotropic effect of the GLE05 found as a difference of two, close to the cutoff rigidity stations Leeds and Chicago.

while the difference in $\mathrm{CR}$ variations at these two stations was negative. During the first $20 \mathrm{~min}$ of the enhancements the anisotropy reached almost $100 \%$ (see Fig. 5) and exceeded the value of $30 \%$ even at 04:30 UT (i.e. $48 \mathrm{~min}$ later the onset).

b) A fast arrival of the first particles is evident just after the onset. Even if we assume that the particle acceleration took place at the very beginning of the flare, the estimated time of the first particle arrival appears to be quite early, indicating that these first protons almost keep up with the light.

c) The maximum flux was reached relatively quickly: in $10-15 \mathrm{~min}$ at favorably located stations (Leeds, Stokholm) whose asymptotic cones of acceptance viewed in the direction of the maximum of the anisotropy flux. At the other stations the intensity peaked approximately in one hour.

d) The energy range of the accelerated particles extended far above $15 \mathrm{GeV}$, although there were no stations with such a rigidity, but this conclusion follows from the magnitude of the enhancement recorded at the stations Huancayo, Mt. Norikura and Mexico $(\mathrm{Rc}=13.45 ; 11.35$ and $9.56 \mathrm{GV}$, correspondingly) and at the muonic detectors and ionization chambers, as well (which are not considered in this paper). The effect as much as $110 \%$ at low latitude NMs and up to 7\% at Tokyo ionization chamber is evidence that $10-15 \mathrm{GeV}$ is not a limit for the solar particles in this flux.

\subsection{Results of modeling}

Several different models have been evaluated by fitting to the neutron monitor data, in order to determine the spectrum and the anisotropy of the solar cosmic rays during this event. 


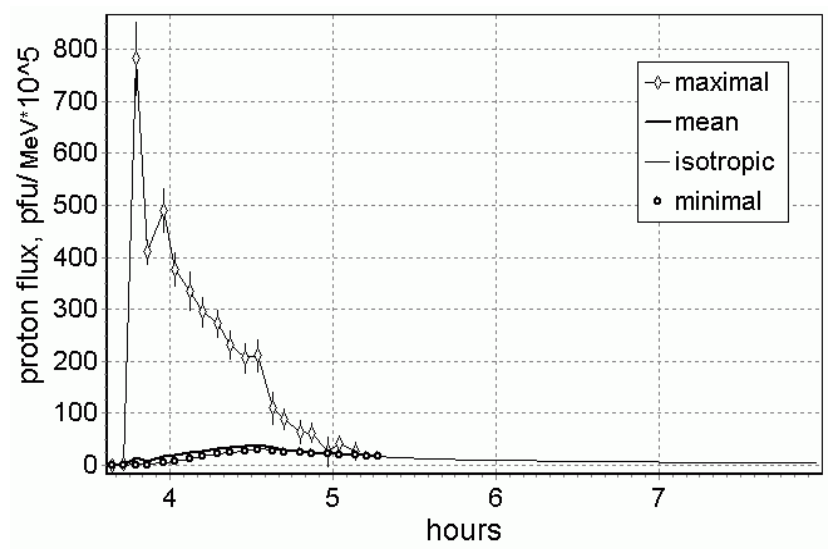

Fig. 6. Behavior of the minimal, maximal, isotropic and mean fluxes of solar cosmic rays of $1 \mathrm{GeV}$ energy at the beginning of the event. Here and in succeeding figures Pfu means the number of particles per $\mathrm{s} . \mathrm{cm}^{2} . s r$

\section{a) Isotropic model}

The simplest isotropic model arises from Eq. (4) when the existence of the second term is ignored. In studying the observational data, one suspects that such an assumption would not be acceptable for the initial stage of enhancement. However, in $1.5 \mathrm{~h}$ after the onset, the anisotropy is sufficiently reduced and therefore the isotropic model can be applied. The isotropic model allows a choice between different energy dependences. We found that the best agreement between observational data and model occurs after having applied a power law spectrum. The residual dispersions differ almost by an order of magnitude in the models with power law and exponential spectra. All power law spectra (in energy or in rigidity) gave almost similar results, however, the best fit was seen for $\Delta I=E^{\gamma}$. Nevertheless, we also tested a more complicated spectrum where the spectral index also depends on energy: $\gamma(E)=\gamma_{0}+\gamma_{1}$.E. The value of $\gamma_{1}$ was found to be very small, hence we decided to use the approximation $\Delta I=E^{\gamma}$ (with $\gamma=\gamma_{0}$ ), leaving a possible application of a more complicated spectrum for the muonioc data case.

\section{b) Anisotropic models}

Assuming an anisotropic model for the cosmic ray intensity variations three different cases of the angular distribution function $\Psi_{1}$ (Eqs. (5a, b, c)) were tested separately. Equation (5a) gave a better fit to the real distribution of the effect than Eq. (5b), while Eq. (5c) gave the best results over all. Thus, for the angular distribution of the solar particle flux we used the approximation $\exp \left(-\left(\mathrm{n}_{a} \sin \left(\chi-\chi_{0}\right)\right)^{2}\right)$ in our further study. The model for the NM counting rate can be written as:

$$
\begin{aligned}
& \left.\frac{\Delta N}{N_{0}}\right|_{i}(t)=b_{0}(t) C_{0 i}\left(E_{i}, h_{i}, \gamma, E_{u}, t\right) \\
& \quad+b_{1}(t) C_{1 i}\left(E_{i}, h_{i}, \gamma, E_{u}, \lambda, \varphi\right)
\end{aligned}
$$

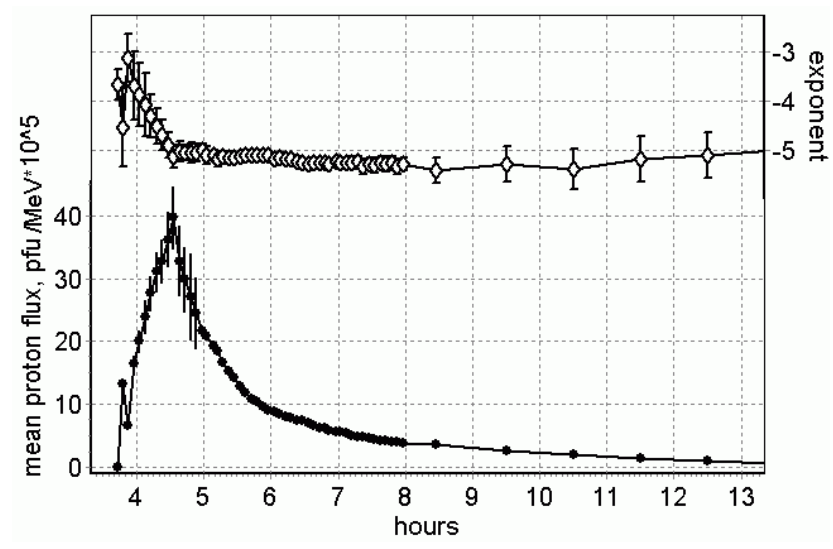

Fig. 7. Variation of the power law index (exponent) in isotropic (diamonds) and anisotropic (circles) models.

with coefficients:

$$
\begin{aligned}
\mathrm{C}_{0 \mathrm{i}}= & \int_{\mathrm{E}_{\mathrm{i}}}^{\mathrm{E}_{\mathrm{u}}} \mathrm{W}\left(\mathrm{E}, \mathrm{t}_{0}, \mathrm{~h}_{\mathrm{i}}\right) \mathrm{E}^{\gamma}(\mathrm{t}, \mathrm{E}) \mathrm{dE} / \int_{\mathrm{E}_{\mathrm{i}}}^{\infty} \mathrm{W}\left(\mathrm{E}, \mathrm{t}_{0}, \mathrm{~h}_{\mathrm{i}}\right) \mathrm{dE} \\
\mathrm{C}_{1 \mathrm{i}}= & \int_{E_{i}}^{E_{u}} W\left(E, t_{0}, h_{i}\right) E^{\gamma}(t, E) \\
& \exp \left(-n_{\alpha}^{2} \sin ^{2}\left(\chi(E)-\chi_{0}\right)\right) / \int_{E_{i}}^{\infty} W\left(E, t_{0}, h_{i}\right) d E,
\end{aligned}
$$

where $b_{0}, b_{1}, \gamma, E_{u}, n_{a}$ and $\chi_{0}$ are time dependent parameters. The first term in Eq. (6) describes an isotropic part and the second one describes the anisotropic portion of the CR variation.

In order to define the position of the anisotropy source, the parameters of latitude $\lambda_{0}$ and longitude $\varphi_{0}$, instead of $\chi_{0}$, are used in Eq. (8). As a result we have seven free parameters, which were calculated by the least-square method. Since the total amount of detectors recording the event was rather small (only 14), we tried to simplify our model by reducing the number of unknown parameters. We utilized exclusively the anisotropic model for the first two 5-min intervals of the event and the isotropic model for the later stage. In other words, for the first two 5-min intervals we took into consideration only the second term of the relation (6) and for the later time we could ignore the anisotropic influence in relation (6) and used only the first term, which allowed the number of unknown parameters to be reduced to three.

The parameters calculated from different models have been used to define the isotropic flux by our isotropic model and the maximal and minimal fluxes by our anisotropic and total models (the results are presented in Fig. 6). Moreover, the mean flux of the solar cosmic rays was also calculated by averaging angular dependences for all directions:

$$
\begin{aligned}
\mathrm{I}_{\text {mean }} & \frac{E) \gamma}{4 \pi}\left(4 \pi b_{0}+b_{1} \int_{0}^{\pi} \exp \left(-n_{a}^{2} \sin ^{2}(\chi(E)-\chi)\right) \sin (\chi) d \chi\right. \\
= & \left(b_{0}+\frac{b_{1}}{n^{2}}\left(1-e^{-n^{2}}\right)\right) E^{\beta}
\end{aligned}
$$




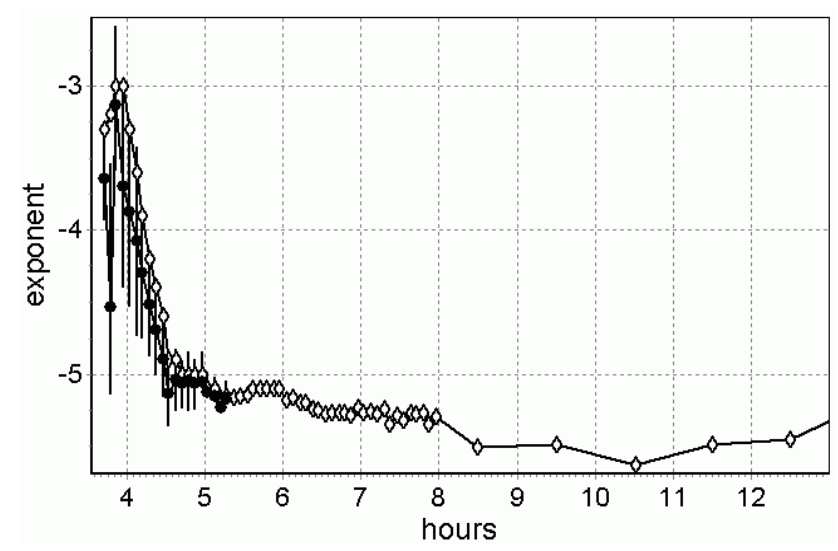

Fig. 8. Behavior of the power law index (exponent) for isotropic (starting from the 16th 5-min interval), and anisotropic (the first 15 five-min intervals) models, together with mean flux, averaged by all directions (for $1 \mathrm{GeV}$ energy).

At the beginning, while the anisotropy is very big, the maximal flux greatly exceeds the minimum and isotropic fluxes, which show similar variation with time. The mean and isotropic fluxes are approximately the same, even during the time of the high anisotropy, and one hour after the onset both fluxes nearly coincide. The maximum flux of the particles arriving in a very narrow beam during the first $15-20 \mathrm{~min}$ exceeds the maximum diffusion flux by a factor greater than 20 . This narrow particle beam is the reason why the 23 February 1956 event was outstanding. The spectral indices derived from different models throughout the event become almost the same starting from the second hour, as is shown in Fig. 7. A difference in the indices exceeds their statistical error only at the very beginning of the event.

Our results, based on the application of a complete model (isotropic plus anisotropic), begin to be less reliable during the second and third hours of the event. This is not surprising since low-latitude stations had not yet observed the enhancement by that time, and we need to define 6-7 model parameters using the data from only 8-9 stations. Additionally, the contribution of the false variations increases along with a decrease in the solar particle effect. So, the most complete model under some conditions turns out to be less reliable. Fortunately, the behavior of the spectral index, as well as the solar CR flux, gives an opportunity to use a simple isotropic model during the later phase of the event. Therefore, in this analysis, we used:

1. a complete anisotropic model for the period 03:40-03:50 UT,

2. a mixed model - for the period 03:50-04:55 UT and

3. an isotropic model, starting from 04:55 UT.

One can see from Fig. 8 that the mean flux of solar cosmic rays, which may be considered as their density, increases

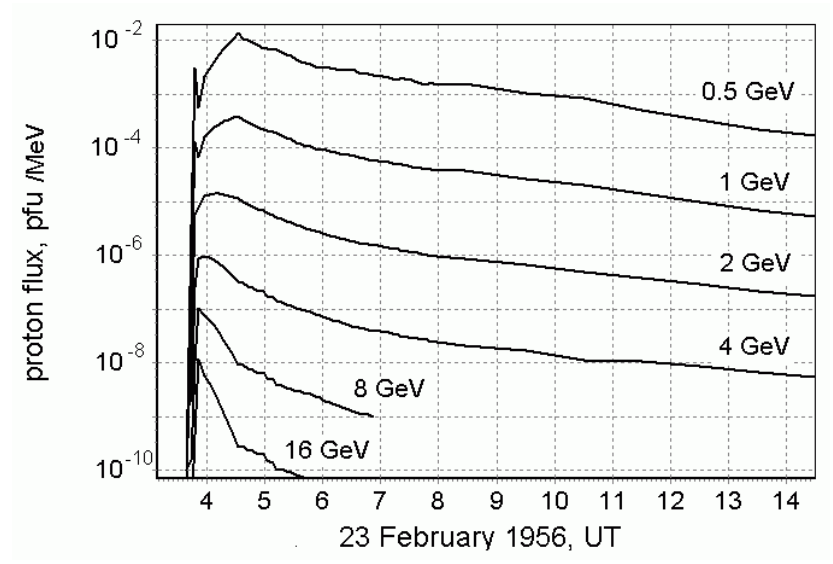

Fig. 9. Differential fluxes of solar protons with energies $0.5,1,2,4$, 8 and $16 \mathrm{GeV}$.

quickly at the beginning of the event and reaches maximum in about $55 \mathrm{~min}: 4 \cdot 10^{-4} \mathrm{~cm}^{-2} \mathrm{~s}^{-1} \mathrm{sr}^{-1}$ for $1 \mathrm{GeV}$ energy. This is only 3 times more than the flux of galactic CR. In the beginning of the event the spectrum of solar cosmic rays is hard: from 03:50 to 03:55 UT the spectral index $\gamma$ reached the value of $-3.1 \pm 0.5$. Throughout the next $45 \mathrm{~min}$ the absolute $\gamma$ value gradually increased and during the rest of the time exceeded 5. Such a behavior of the spectral index is typical for a GLE. An analogous time evolution of $\gamma$ was also observed during the GLE on September 1989 (Baisultanova et. al., 1990, 1992; Smart and Shea, 1991). After 11:00 UT the spectrum tends to harden but this result appears not to be reliable since the solar CR flux at that time became small and comparable with the statistical variations in the baseline neutron monitor counting rate.

The solar CR differential flux was calculated for different energies employing the mean flux variations and spectral index presented in Fig. 8. The results are plotted in Fig. 9. It is clearly seen from this figure that the peak flux time $t_{\max }$ depends on the energy of the cosmic ray particles. We applied a diffusion model for the cosmic particle propagation, assuming that $\mathrm{t}_{\max }$ is proportional to the inverse effective diffusion coefficient $k$, which depends on the energy as $\kappa(E) \propto E^{\alpha}$. The value of the $\alpha$ index within the energy range $2-8 \mathrm{GeV}$ was obtained as $\alpha=0.6 \pm 0.2$. Consequently, if we are to judge from the neutron monitor data, the proton enhancement near Earth for $16 \mathrm{GeV}$ (and even for $8 \mathrm{GeV}$ ) was very short in duration.

Drawing this conclusion we have to take into account that the upper energy limit $\mathrm{E}_{u}$ for the accelerated particles cannot be found reliably in this event from the neutron monitor data. The maximal cutoff rigidity at which the enhancement is still observed at a certain instant (Fig. 10), gives a lower limit for the $\mathrm{E}_{u}$, but varying $\mathrm{E}_{u}$ in the higher energy region gives very weak changes in the residual dispersion that leads to uncertainty in the $\mathrm{E}_{u}$ definition. It means that even a rather large upper limit of energy for accelerated particles (for example, $100 \mathrm{GeV}$ ) does not contradict the neutron monitor 


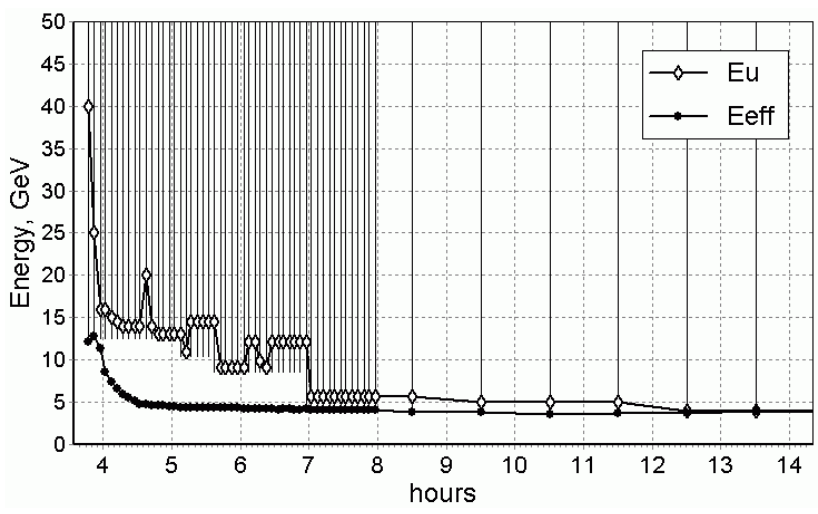

Fig. 10. Behavior of upper limited energy $\mathrm{E}_{u}$ of solar CR (diamonds) and their effective energy $\mathrm{E}_{e f f}$ from neutron monitor network registration.

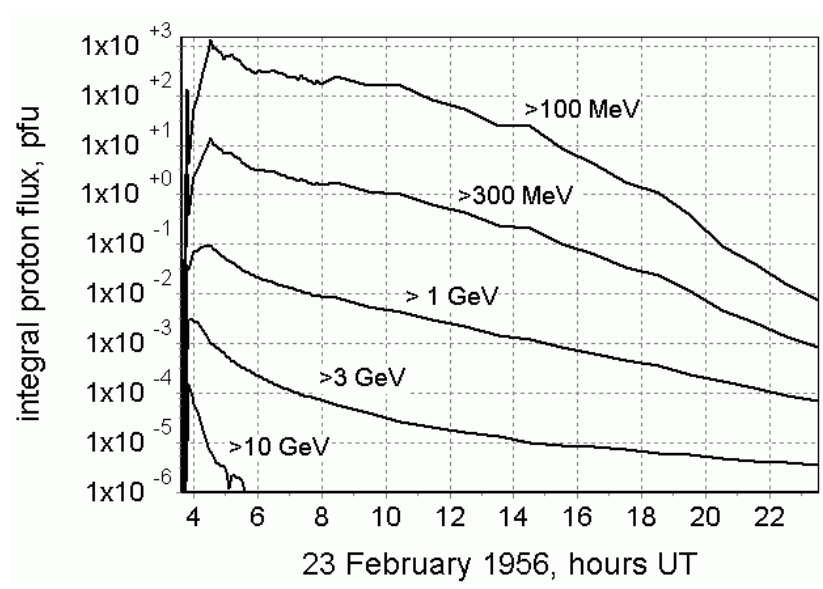

Fig. 11. Integral flux of protons with energies $>100 \mathrm{MeV}$, $>300 \mathrm{MeV},>1,>3$ and $>10 \mathrm{GeV}$.

data. Thus, $\mathrm{E}_{u}$ turns out to be the least meaningful parameter among all calculated, and therefore it can be reasonable specified in advance without a big loss in the accuracy of the other parameters. The uncertainty in the measurement of $\mathrm{E}_{u}$ is a peculiarity of this event in which solar CR were accelerated up to unusually high energies.

The upper energy $\mathrm{E}_{u}$, as well as the effective energy $\mathrm{E}_{e f f}$ for solar cosmic rays recorded by neutron monitors, are presented in Fig. 10. The effective energy for each separate detector is defined as the energy $\mathrm{E}_{e i}$ at which the density variation $\mathrm{I}_{\text {mean }}(\mathrm{E})$ of the primary solar cosmic rays is equal to the counting rate variation $\delta_{i}$. In the case of a power law spectrum:

$$
\ln \left(E_{e i}\right)=\gamma^{-1}\left(\ln \left(\delta_{i}\right)-\ln \left(I_{\text {mean }}(1 \mathrm{GeV})\right)\right)
$$

The effective energy $\mathrm{E}_{\text {eff }}$ for the whole neutron monitor network has been calculated for each time interval by averaging $\mathrm{E}_{e i}$ over the neutron monitor network with the weighting function assumed to be equal to the magnitude of the effect

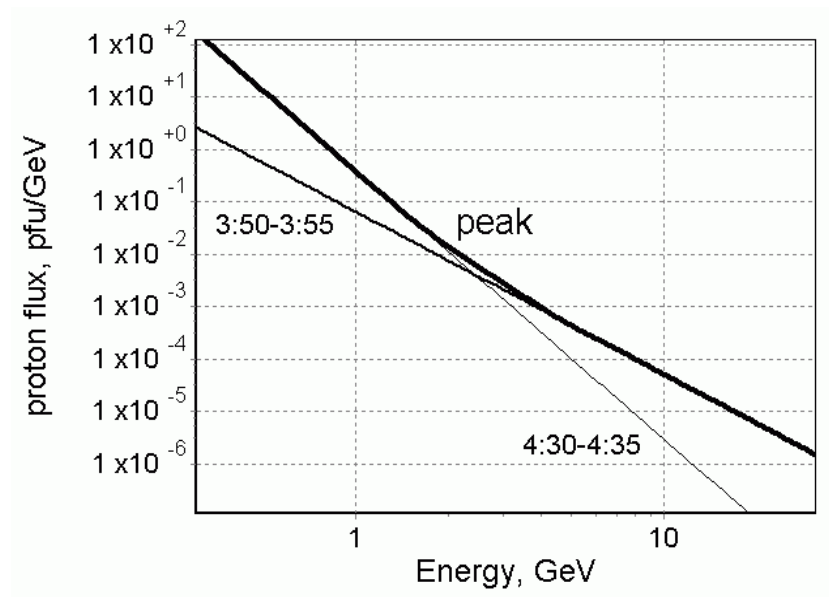

Fig. 12. Energy spectra of the CR mean flux at two different moments of enhancement and peak spectrum for GLE 23 February derived from the neutron monitor data.

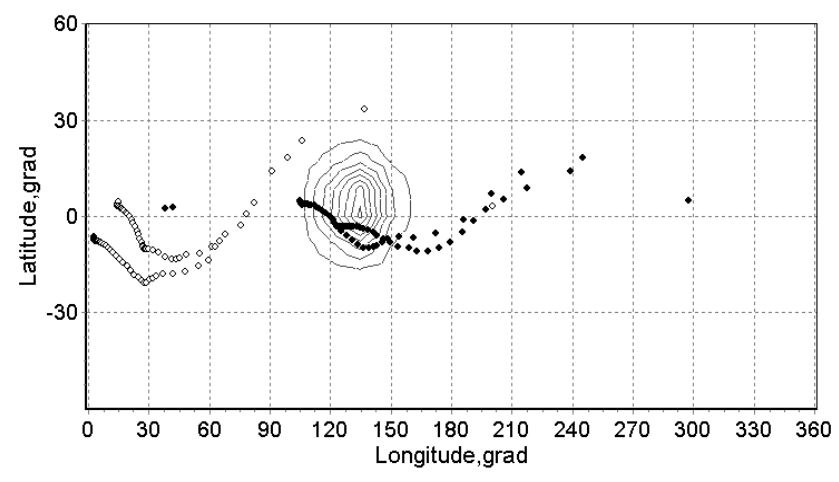

Fig. 13. Location of the solar CR anisotropy source at 03:4003:50 UT (isometric curves of the equal fluxes) and asymptotic directions of vertical incident particles with energy $<15 \mathrm{GeV}$ for two stations (Gottingen and Leeds) with maximal effect (black points) and two stations (Ottawa and Chicago) with zero effect at this time (opened circles).

$\delta_{i}$ at each NM from solar cosmic rays:

$\mathrm{E}_{\mathrm{eff}}=\sum_{i=1}^{n} E_{e i} \delta_{i} / \sum_{i=1}^{n} \delta_{i}$

The effective energy seems to be high only at the beginning of the event. In less than one hour it decreased to 4$5 \mathrm{GeV}$. In the later phase of the enhancement the values $\mathrm{E}_{\text {eff }}$ and $E_{\mathrm{u}}$ became almost the same. Near $\mathrm{E}_{\text {eff }}$ the energy spectra slope can be obtained with the best accuracy.

In Fig. 11 the behavior of integral fluxes of the solar CR is presented. The rapid drop in the high energy particle flux is more evident in the integral presentation than in the differential. The results displayed in Fig. 11 for energies greater than $300 \mathrm{MeV}$ and $100 \mathrm{MeV}$ are, of course, derived by extrapolation. No neutron monitor in 1956 could record cosmic ray particles with energies $<500 \mathrm{MeV}$ and dependences for low energy cosmic rays are obtained with the assumption that the 


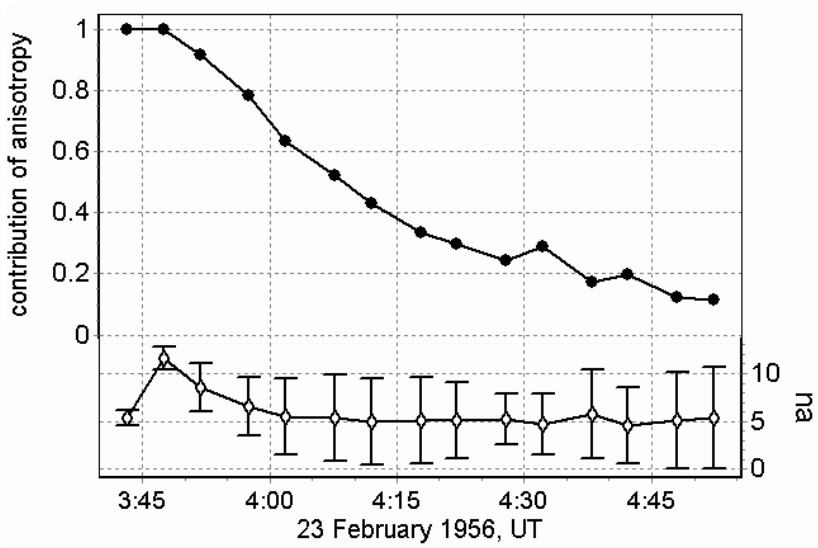

Fig. 14. Behavior of the anisotropy contribution A part and coefficient $\mathrm{n}_{a}$, characterizing a width of angular distribution of the solar CR anisotropic flux.
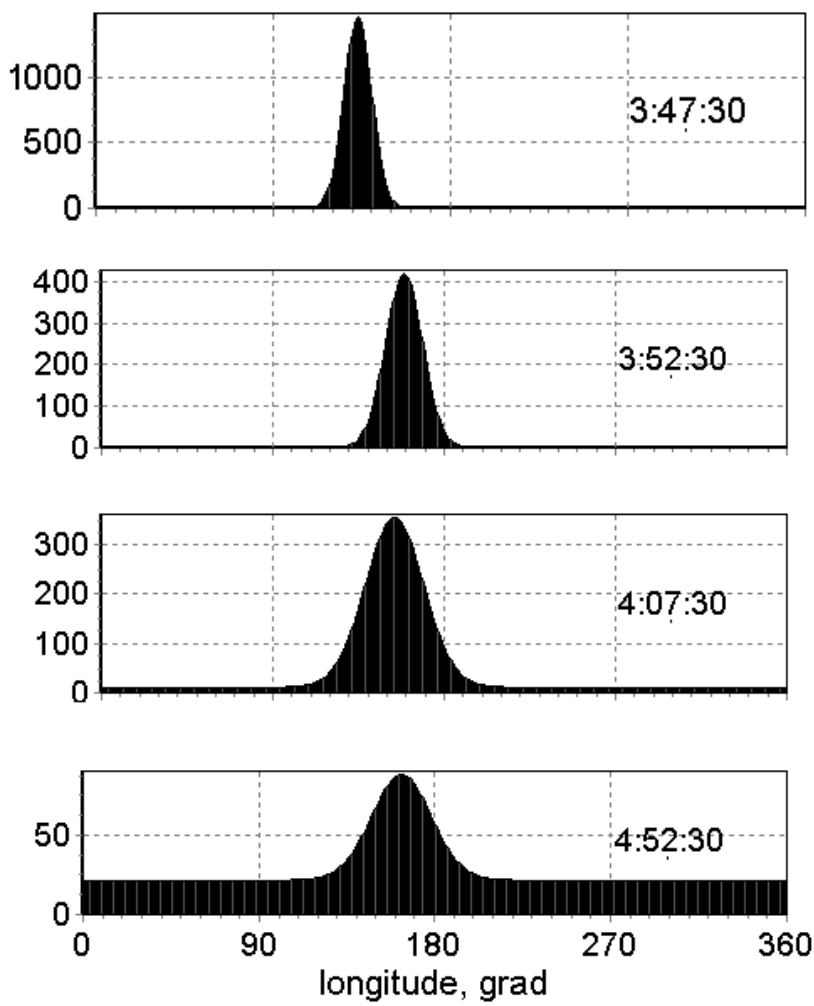

Fig. 15. Dynamic of the longitudinal distribution of the solar cosmic ray flux at the Earth's equator during the first $75 \mathrm{~min}$ of the enhancement. Along the ordinate axis the flux of solar cosmic rays is plotted in $\mathrm{pfu} / \mathrm{MeV} * 10^{5}$.

spectra shape and the spectral index $\gamma$ are independent of energy. Nevertheless, it would be interesting to compare the estimations obtained with some real onboard measurements by IMP- 8 and GOES. The biggest fluxes for $>100 \mathrm{MeV}$ protons (about $600 \mathrm{pfu}$ ) were recorded on 29 September 1989 and on 14 July 2000. Thus, the estimated flux for $>100 \mathrm{MeV}$ particles in February 1956 exceeds by only a factor of two

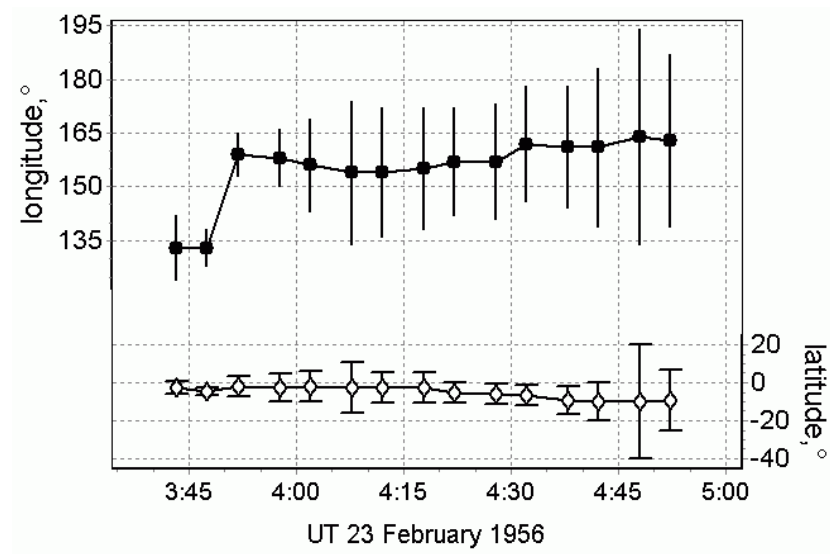

Fig. 16. Changes in the geographic longitude and latitude of the anisotropy source along the event duration.

the observed maximal values in the satellite epoch. Having worked out time and magnitude of the peak fluxes for different energies we obtained the peak spectrum within the energy range from $500 \mathrm{MeV}$ to $25 \mathrm{GV}$ (Fig.12) - the energy dependence of the maximum fluxes of solar protons.

In the diffusion approximation the peak spectrum corresponds to the release spectrum in the source, which is not distorted with the particle propagation. As we see, it is quite close to a power law spectrum. Within the energy range 2.7$4.5 \mathrm{GeV}$ the spectral index is $4.51 \pm 0.13$. Some hardening in the high energy range may be due to a contribution to the maximum fluxes of high energy protons, which came without scattering. At the beginning of the event the particles arrived in a very narrow beam. In Fig. 13 the position of the particles' source $\left(\mathrm{n}_{a}=8\right)$ at 03:40-03:50 UT is presented, together with the asymptotic directions for those stations which observed the largest effect and the zero effect. The outward curve corresponds to a flux of about $10 \%$ of the maximum in the assumption that the angular dependence $n_{a}$ in Eq. $(5 \mathrm{c})$ is equal to 8. As it follows from Fig. 13 the directions with maximum and minimum effect are close to each other at the longitude $\sim 100^{\circ}$. If the angle distribution turned out to be even 1.5 wider, the effect would be recorded at all stations. How narrow was this particle beam? This is one more question which could be answered by means of the data from ionization chambers and muonic telescopes operating at that time.

The contribution of the anisotropic part of solar cosmic ray flux to the total flux from all directions was calculated by:

$A_{\text {part }}=\frac{b_{1}}{b_{0} n_{a}^{2}}\left(1-e^{-n_{a}^{2}}\right)$.

This parameter is shown in Fig. 14, together with parameter $\mathrm{n}_{a}$ controlling the width of the angular distribution. As clearly seen from Fig. 14, the anisotropy dominates during the first $30 \mathrm{~min}$ of the effect and then decreases quickly with time. The narrowest beam is observed at the beginning of the effect and at that exact time the data certainly 
contradict the assumption of a wide angular distribution, although this assumption becomes more acceptable with time. The time evolution of the angular distribution is presented in Fig. 15. Next, Fig. 16 illustrates the time-dependent variation of the position of the source of the solar particle anisotropy near Earth in geographic coordinates. The source of the anisotropy (direction to the flux maximum) was located close to the ecliptic plane and in the same quadrant as the nominal IMF. A sharp change in the source longitude $\left(\sim 23^{\circ}\right) 10 \mathrm{~min}$ after the onset stands out in Fig. 16. This cannot be attributed to a difference between the models, although at this moment we replaced an anisotropic model by a mixed one. Moreover, this longitudinal jump also appears even if we do not change the model. It is noteworthy to say that at the same moment the essential changes in the energetic spectrum occurred, and only $15 \mathrm{~min}$ after the onset the spectrum started to soften gradually. Of course, the anomaly in the parameters' behavior during the first 10-15 min could also be attributed to the quality of the interpolated data; however, we think that it is evidence of a real anomaly in the solar particles' behavior.

Since the flare and assumed source of the particle acceleration were located far to the west from the Sun-Earth line, it is natural to expect that charged particles reached the force lines that were erathward after some delay. Perhaps they came to these lines after diffusion transversely on the field over $\sim 10$ $15 \mathrm{~min}$. The particle flux focused along our force line began to dominate only after the third 5-min interval. Afterwards the source of anisotropy was placed between $154^{\circ}$ and $164^{\circ}$ longitudes, which corresponds to the direction along the classic Archimedean spiral path for a high solar wind velocity (Smart and Shea, 1990). But the first, most energetic particles were on our force line near the Earth's orbit, so their source was seen as more western. This subject of course is still unanswered but we hope to obtain some more reliable conclusions after employing the data from muonic detectors and ionization chambers.

\section{Summary}

The worldwide neutron monitor network in its modern configuration began to be created during the International Geophysical Year - 1957. Nevertheless, several neutron detectors were operating in 1956, although not all in the modern standard configuration, and were sufficient to obtain the main characteristics and dynamics of the 23 February 1956 ground level enhancement of solar cosmic rays.

The results of our analysis are in agreement with Smart and Shea (1990), complementing and extending them. The most outstanding feature of this proton enhancement was a narrow and extremely intensive beam of ultra-relativistic particles arriving at Earth during the first minutes of the event. The neutron monitors whose asymptotic directions viewed the anisotropy source (to meet the stream) recorded an enhancement of thousands of percents. None of the succeeding 64 GLEs (perhaps except for the last GLE in January 2005) gave amplitudes in cosmic ray variations comparable with this effect. However, this unique beam was observed during the short time and its width did not exceed $30-40^{\circ}$. Thus, its contribution to the solar particle density, as well as to their fluency, was not very significant.

Although the estimation of the integral flux for particles with energies graeter than $100 \mathrm{MeV}$ ranks this event above all succeeding, nevertheless, it does not drop out from the common distribution. For the particles of $>10 \mathrm{MeV}$ energy (if to prolong an extrapolation) this event appears not to be unique but rather one of the largest proton enhancements. This is in fairly good agreement with riometer data (Shea and Smart, 1990), on which the effect during the more recent events was even stronger than in February 1956. Perhaps the number of accelerated low energy particles also reached a record figure, but these particles passed mainly to the west of the Earth.

Many features of this GLE apparently may be explained by the peculiarity of the particle interplanetary propagation from a remote (limb or behind the limb) source.

The quality of the data which we used, although allowing us to extract much more information on this event, does not give full confidence in some details: the unusual changes in the anisotropy characteristics; the energy spectrum during the first 15 min of the enhancement; the spectra changes during the late stage of the event and also the spectra hardening with increasing energy. A joint analysis of data from NMs, ionization chambers and muonic telescopes will possibly lead to more reliable answers as to whether these changes are real or not.

Acknowledgements. Thanks are due to all colleagues who kindly provided Neutron Monitor data. This work is partly supported by FBR Russian grant 04-02-16763; by Greece program ENTER 2001 (grant 70/3/6890) and IRAKLITOS (grant 70/3/7218). We are very appreciated to R. Pyle for his help with this paper writing.

Topical Editor R. Forsyth thanks K. Kudela and J. F Valdés Galicia for their help in evaluating this paper.

\section{References}

Akasofu, S.-I. and Chapman, S.: Solar-terrestrial physics, Clarendon press, Oxford, 1972.

Baisultanova, L. M., Belov, A. V., Gushchina, R. T., Dorman, L. I., Eroshenko, E. A., Ishkov, V. N., and Yanke, V. G.: Preliminary analysis of the solar cosmic ray enhancement on 29 September 1989 by the neutron monitor data (in Russian), Preprint 24(909), IZMIRAN, 1990.

Baisultanova, L. M., Belov, A. V., Dorman, L. I., Eroshenko, E. A., and Gushchina, R. T.: The features of the ground level increase of cosmic ray intensity on September 29, 1989, Proc. of the 1-st SOLTIP Symposium, Liblice, 2, 7-13, 1992.

Belov, A. V.: Large scale modulation: view from the Earth, Space Sci. Rev., 93, 1, 79-105, 2000.

Belov, A. V. and Eroshenko, E. A.: The energy spectra and other properties of the great proton events during 22-nd solar cycle, Adv. Space Res., 17, 167-170, 1996. 
Belov, A. V. and Struminsky, A. B: Neutron monitor sensitivity to primary protons below $3 \mathrm{GeV}$ derived from data of ground level events, Proc. of the 25-th ICRC, 1, 201-204, 1997.

Belov, A. V., Eroshenko, E. A., and Livshits, M. A.: The Energy Spectra of the Accelerated Particles Near the Earth and in the Source in 15 June 1991 Enhancement, Proc. of the 8-th Symp. on Solar Terrestrial Physics, Sendai, Japan, 26-29, 1994.

Belov, A. V., Gushchina, R. T., and Yanke, V. G.: Long term cosmic ray variations: spectrum and relation with solar activity, Proc. 25-th ICRC, 2, 61-64, 1997.

Belov, A., Eroshenko, E., Mavromichalaki, H., Plainaki, C., and Yanke, V.: A study of the ground level enhancement of 23 February 1956, Adv. Space Res., 35, 697-701, 2005.

Clem, J. and Dorman, L. I.: Neutron monitor response functions, Space Sci. Rev., 93, 1, 335-359, 2000.

Dorman, L. I.: Variatsii Kosmicheskix Luchej (Gostexizdat, Moscow 1957), English translation (limited edition): Cosmic Ray Variations (Technical liaison office, Wright-Patterson Air Force Base, Ohio), 1957.

Dorman, L. I.: Progress in Elementary Particles and Cosmic Ray Physics, edited by: Wilson, J. G. and Wouthuysen, S. A., NorthHolland Publ. Co., Amsterdam, 1963.

Dorman, L. I. and Miroshnichenko, L. I.: Solar Cosmic Rays, Moscow: Nauka (in Russian), 468, 1968.

Heristchi, Dj., Trottet, G., and Pererez-Peraza, J.: Upper cutoff of High energy Solar Protons, Solar Phys., 49, 151-175, 1976.

Meyer, P., Parker, E. N., and Simpson, J. A.: Solar cosmic rays of February 1956 and their propagation through interplanetary space, 104, 3, 768-783, 1956.

Miroshnichenko, L. I.: On the absolute fluxes of particles accelerated at the Sun on February 23, 1956, Geomagn. and Aeronomy, 10, 5, 898-900, 1970.

Miroshnichenko, L. I.: Solar Cosmic Rays, Kluwer Academic publishers, 492, 2001.

Moraal, H., Belov, A., and Clem, J. M.: Design and co-ordination of multi-station international neutron monitor networks, Space Sci. Rev., 93, 285-303, 2000

Pfotzer, G.: On the separation of direct and indirect fractions of solar cosmic radiation on February 23, 1956 and on difference in steepness of momentum spectrum of these two components, Nuovo Cimento Suppl., 8, Series 10, No. 2, 180-187, 1958.
Sandstrom, A. E.: Cosmic Ray Physics, North-Holland publishing company-Amsterdam, 1965.

Sarabhai, V., Duggal, S. P., Razdan, H., and Sastry, F. S. G.: A solar flare type increase in cosmic rays at low latitudes, Proc. Ind. Acad. Sci., 43, 309-318, 1956.

Shea, M. A. and Smart, D. F.: A five by fifteen degree world grid of calculated cosmic ray cutoff rigidities for 1965 and 1975, Proc. of 14th ICRC 4, 1298-1303, 1975.

Shea, M. A. and Smart, D. F.: Fifty years of cosmic radiation data, Space Sci. Rev., 93, 229-262, 2000.

Shea, M. A. and Smart, D. F.: Solar proton event patterns: the rising portion of five solar cycles, Adv. Space Res., 29 3, 325-330, 2002.

Shea, M. A., Smart, D. F., and McCracken, K. G.: A Study of Vertical Cutoff Rigidity Using Sixth Degree Simulations of the Geomagnetic Field, J. Geophys. Res., 70, 4117-4130, 1965.

Smart, D. F. and Shea, M. A.: Probable pitch angle distribution and spectra of the 23 February 1956 solar cosmic ray event, Proc. 21st Int. Cosmic Ray Conf., Adelaide, Australia, 5, 257-260, 1990.

Smart, D. F. and Shea, M. A.: A comparison of the magnitude of the 29 September 1989 high energy event with solar cycle 17, 18 and 19 events, Proc. 22nd Int. Cosmic Ray Conf., Dublin, Ireland, 3, 101-104, 1991.

Stoker, P. H., Van der Walt, A. J., and Potgieter, M. S.: Modulation of cosmic rays during solar minimum, Cosmic ray intensity survey at sea-level during 1976: Experimental details, S. Afr. J. Phys. 3, 73-76, 1980.

Van Allen, J. A. and Winckler, J. R.: Spectrum of low-rigidity cosmic rays during the solar flare of February 23, 1956, Phys. Rev., 106, 1072-1073, 1957.

Villoresi, G., Dorman, L. I., Iucci, N., and Ptitsyna, N. G.: Cosmic ray survey to Antarctica and coupling functions for neutron component near solar minimum (1996-1997), 1. Methodology and data quality assurance, J. Geophys. Res., 105, A9, 21 025$21034,2000$.

Yasue, S., Mori, S., Sakakibara, S. and Nagashima, K.: Coupling coefficients of Cosmic Ray Daily Variations for Neutron Monitor Stations, Report of Cosmic-Ray Research Laboratory, N7, Nagoya, Japan, 1982. 